lustitia Socialis. Revista Arbitrada de Ciencias Jurídicas.

Año V. Vol. V. №3. Edición Especial. 2020-III:

Universidad Regional Autónoma de los Andes

Hecho el depósito de Ley: FA2016000064 ISSN: 2542-3371

FUNDACIÓN KOINONIA (F.K). Santa Ana de Coro, Venezuela

Paul Alejandro Centeno-Maldonado; Alex Carlos Ortega-Sinche; Williams Rafael Santillán-Abarca

http://dx.doi.org/10.35381/racii.v5i3.1109

\title{
Criterio normativo aplicable en defraudación tributaria en el Ecuador
}

\section{Regulatory criteria applicable to tax fraud in Ecuador}

\author{
Paul Alejandro Centeno-Maldonado \\ ur.paulcenteno@uniandes.edu.ec \\ Universidad Regional Autónoma de los Andes, Riobamba \\ Ecuador \\ https://orcid.org/0000-0001-6541-8078 \\ Alex Carlos Ortega-Sinche \\ acortegauniandesr@gmail.com \\ Universidad Regional Autónoma de los Andes, Riobamba \\ Ecuador \\ Williams Rafael Santillán-Abarca \\ rafa21santillan@gmail.com \\ Universidad Regional Autónoma de los Andes, Riobamba \\ Ecuador
}

Recibido: 31 de octubre de 2020

Revisado: 10 de octubre de 2020

Aprobado: 05 de diciembre de 2020

Publicado: 10 de diciembre de 2020 
lustitia Socialis. Revista Arbitrada de Ciencias Jurídicas.

Año V. Vol. V. №3. Edición Especial. 2020-III:

Universidad Regional Autónoma de los Andes

Hecho el depósito de Ley: FA2016000064

ISSN: 2542-3371

FUNDACIÓN KOINONIA (F.K). Santa Ana de Coro, Venezuela

Paul Alejandro Centeno-Maldonado; Alex Carlos Ortega-Sinche; Williams Rafael Santillán-Abarca

\title{
RESUMEN
}

La investigación tiene por objetivo analizar el criterio normativo aplicable en defraudación tributaria en el Ecuador. Se desarrolló metodológicamente desde un tipo descriptiva documental con diseño bibliográfico, lo cual permitió a través del método analítico sintético y de la técnica de análisis de contenido. Se evidencia que existe mejora en la configuración de un sistema de recaudación automatizado que coopera en la recolección de tributos, así como de crear una cultura para el pago de impuestos, sin embargo, el sistema es frágil en cuanto a los procesos aplicados para sancionar fuertemente a quienes incurran en delitos o fraudes fiscales, por lo tanto, se considera como necesaria la igualdad tributaria desde una reconstrucción de la jurisprudencia, siendo un tema a debatir por la comunidad jurídica del Ecuador con la finalidad de optimizar lo referido a la tributación en todos sus niveles.

Descriptores: Tributación; política fiscal; derecho administrativo. (Palabras tomadas del Tesauro UNESCO).

\begin{abstract}
The objective of the investigation is to analyze the normative criteria applicable to tax fraud in Ecuador. It was methodologically developed from a descriptive documentary type with bibliographic design, which allowed through the analytical-synthetic method and the content analysis technique. It is evidenced that there is an improvement in the configuration of an automated collection system that cooperates in the collection of taxes, as well as creating a culture for the payment of taxes, however, the system is fragile in terms of the processes applied to strongly sanction to those who commit crimes or tax fraud, therefore, tax equality is considered necessary from a reconstruction of jurisprudence, being an issue to be debated by the legal community of Ecuador in order to optimize what refers to taxation in all your levels.
\end{abstract}

Descriptors: Taxation; fiscal policy; administrative law. (Words taken from the UNESCO Thesaurus). 
lustitia Socialis. Revista Arbitrada de Ciencias Jurídicas.

Año V. Vol. V. N³. Edición Especial. 2020-III:

Universidad Regional Autónoma de los Andes

Hecho el depósito de Ley: FA2016000064

ISSN: 2542-3371

FUNDACIÓN KOINONIA (F.K). Santa Ana de Coro, Venezuela

Paul Alejandro Centeno-Maldonado; Alex Carlos Ortega-Sinche; Williams Rafael Santillán-Abarca

\section{INTRODUCCIÓN}

Los procesos administrativos o penales en materia tributaria se dan con una frecuencia considerable, pero es evidente la omisión que por acción debidos a que los servidores en cuanto a declaraciones para poder desviar los fondos a cuentas particulares, la falta de criterio normativo es un gran aliado para los infractores debido a que existen muchas inconsistencias y vacíos legales (Chávez, 2015). Las sanciones de delitos de defraudación tributaria son muy frágiles en cuanto a los procedimientos innecesarios e incluso poco prósperos procedimentalmente, en muchos casos servidores públicos competentes para estos procedimientos mencionados anteriormente no actúan correctamente y conforme a derecho corresponde por la falta de capacitación y experticia en el ámbito tributario. (Sánchez-Huete, 2016).

En este sentido, (De-La-Torre-Lascano, 2017), considera que existe una frágil interpretación de la normativa sobre la tributación y su evasión, por cuanto existe controversia doctrinal sobre lo que se considera como fraude tributario, evasión, lavado de capitales, configurando un accionar jurídico en procura de promover sanciones ejemplares. En Ecuador, el criterio normativo sobre los ilícitos tributarios es un gran problema debido a que no se interpreta y se aplica de manera objetiva la normativa correspondiente en el país haciendo referencia esencialmente al Código Tributario, Código Orgánico Integral Penal y afines los cuales aplican sanciones a los ilícitos tributarios entre ellas la defraudación tributaria, faltas reglamentarias, contravenciones siempre y cuando se cumplan presupuestos establecidos para cada conducta.

En relación de proseguir estudiando el tema en el Ecuador, (Sánchez-Fonseca, et al., 2020), plantean que el sistema de recaudación ha mejorado por cuanto se ha automatizado, sin embargo, se evidencia escasa cultura para pagar los tributos como es debido, aun, cuando se expiden los recibos en las compras, lo cual alerta sobre la necesidad de educar a la ciudadanía para actuar conforme a lo planteado por la ley con la intención de evitar fraudes tributarios, por otro lado, (Saltos-Orrala, 2017), destaca que las leyes tributarias en el Ecuador, tienen su fundamento en la Constitución de la 
lustitia Socialis. Revista Arbitrada de Ciencias Jurídicas.

Año V. Vol. V. N³. Edición Especial. 2020-III:

Universidad Regional Autónoma de los Andes

Hecho el depósito de Ley: FA2016000064

ISSN: 2542-3371

FUNDACIÓN KOINONIA (F.K). Santa Ana de Coro, Venezuela

Paul Alejandro Centeno-Maldonado; Alex Carlos Ortega-Sinche; Williams Rafael Santillán-Abarca

República de 2008, lo cual ventila que existe actualización constitucional, siendo importante revisar si las normativas, procedimientos, se encuentran fundamentos en tal figura, pero sobre todo, estudiar el tema de las sanciones a los infractores, por cuanto, si estas son concebidas como frágiles o de poco alcance sancionatorio, se generará una perspectiva favorable hacia el fraude fiscal.

En razón de lo planteado, la investigación tiene por objetivo analizar el criterio normativo aplicable en defraudación tributaria en el Ecuador.

\section{MÉTODO}

La investigación se desarrolló metodológicamente desde un tipo descriptiva documental con diseño bibliográfico, lo cual permitió a través del método analítico - sintético y de la técnica de análisis de contenido, con la finalidad de escrutar información relacionada al tema de estudio, ubicada en leyes, normas, investigaciones, con la finalidad de sustraer las ideas de mayor realce con la finalidad de ser interpretadas, construyendo una síntesis teórica en conformidad de responder al objetivo del actual trabajo investigativo.

\section{RESULTADOS}

Se destaca que la normativa sancionatoria en materia de evasión tributara anteriores a la designación del Código Orgánico Integral Penal en 2014, se encontraba distribuida para su aplicación en diversos códigos, lo cual generaba un distanciamiento con lo penal, es decir, antes del 2014 no se consideraban como parte penal para ser sancionada como tal, se destaca que el derecho tributario antes del 2014, consideraba como infracciones tributarias, lo siguiente:

a) Delitos,

b) Contravenciones,

c) Faltas reglamentarias.

Sin embargo, con la puesta en vigencia del Código Orgánico Integral Penal 2014, se rige por lo fijado en el artículo 341, donde se promueve: 
lustitia Socialis. Revista Arbitrada de Ciencias Jurídicas.

Año V. Vol. V. N³. Edición Especial. 2020-III:

Universidad Regional Autónoma de los Andes

Hecho el depósito de Ley: FA2016000064 ISSN: 2542-3371

FUNDACIÓN KOINONIA (F.K). Santa Ana de Coro, Venezuela

Paul Alejandro Centeno-Maldonado; Alex Carlos Ortega-Sinche; Williams Rafael Santillán-Abarca

a) El delito: procedimiento establecido en el Código Orgánico Integral Penal,

b) Las Contravenciones y Faltas Reglamentarias: procedimiento establecido en el Código Tributario (Código Tributario, 2005).

En este sentido, en la actualidad, existen dos modalidades de pena tributaria en el Ecuador, la primera de orden penal, calificada como delito, la segunda, como infracciones, faltas, tipificadas en el Código Tributario 2005, destacándose que este es anterior a la Constitución de la República de 2008, lo que obliga a su revisión, adecuación, con la finalidad de evitar contraposiciones constitucionales en la aplicación de la ley que deriven en detrimento de sanciones conformes a generar cambios en los contribuyentes en favor de promover acciones apegada a la ética para la contribución fiscal.

Tabla1.

Artículos o normas sobre la tributación en Ecuador.

\begin{tabular}{|c|c|}
\hline Código Tributario 2005 & tegral \\
\hline $\begin{array}{l}\text { Art. 348.- Concepto. - Son } \\
\text { contravenciones tributarias, las acciones } \\
\text { u omisiones de los contribuyentes, } \\
\text { responsables o terceros o de los } \\
\text { empleados o funcionarios públicos, que } \\
\text { violen o no acaten las normas legales } \\
\text { sobre administración o aplicación de } \\
\text { tributos, u obstaculicen la verificación o } \\
\text { fiscalización de los mismos, o impidan o } \\
\text { retarden la tramitación de los reclamos, } \\
\text { acciones o recursos administrativos. }\end{array}$ & $\begin{array}{l}\text { La persona que simule, oculte, omita, falsee } \\
\text { o engañe a la Administración Tributaria } \\
\text { para dejar de cumplir con sus obligaciones } \\
\text { o para dejar de pagar en todo o en parte los } \\
\text { tributos realmente debidos, en provecho } \\
\text { propio o de un tercero }\end{array}$ \\
\hline $\begin{array}{l}\text { Art. 351.- Concepto. - Son faltas } \\
\text { reglamentarias en materia tributaria, la } \\
\text { inobservancia de normas reglamentarias } \\
\text { y disposiciones administrativas de } \\
\text { obligatoriedad general, que establezcan } \\
\text { los procedimientos o requisitos } \\
\text { necesarios para el cumplimiento de las } \\
\text { obligaciones tributarias y deberes } \\
\text { formales de los sujetos pasivos. }\end{array}$ & $\begin{array}{l}\text { Art. } 298 \text { numeral } 1 \text { del Código Orgánico } \\
\text { Integral Penal: Utilice identidad o } \\
\text { identificación supuesta o falsa en la } \\
\text { solicitud de inscripción, actualización o } \\
\text { cancelación de los registros que llevan las } \\
\text { administraciones } \\
\text { Art. } 298 \text { numeral } 2 \text { del Código Orgánico } \\
\text { Integral Penal: Utilice datos, información o } \\
\text { documentación falsa o adulterada en la }\end{array}$ \\
\hline
\end{tabular}


lustitia Socialis. Revista Arbitrada de Ciencias Jurídicas.

Año V. Vol. V. N³. Edición Especial. 2020-III:

Universidad Regional Autónoma de los Andes

Hecho el depósito de Ley: FA2016000064 ISSN: 2542-3371

FUNDACIÓN KOINONIA (F.K). Santa Ana de Coro, Venezuela

Paul Alejandro Centeno-Maldonado; Alex Carlos Ortega-Sinche; Williams Rafael Santillán-Abarca

\begin{abstract}
Artículo 96 del Código Tributario, en concordancia con el artículo 20 de la Ley de Régimen Tributario Interno, establece como deber formal de los sujetos pasivos llevar los libros y registros contables relacionados con la correspondiente actividad económica, en idioma castellano; anotar, en moneda de curso legal, sus operaciones 0 transacciones y conservar tales libros y registros, mientras la obligación tributaria no esté prescrita; el no llevar estos libros y registros es penable como contravención cuya sanción pecuniaria es una multa de US $\$ 30,00$ a US $\$$ 1.500,00 dólares de los Estados Unidos de América
\end{abstract}

solicitud de inscripción, actualización o cancelación de los registros que llevan las administraciones tributarias.

Art. 298 numeral 3 del Código Orgánico Integral Penal: Realice actividades en un establecimiento a sabiendas de que se encuentre clausurado

Art. 298 numeral 4 del Código Orgánico Integral Penal: Imprima o haga uso de comprobantes de venta o de retención o de documentos complementarios que no sean autorizados por la Administración Tributaria.

Art. 298 numeral 5 del Código Orgánico Integral Penal: Proporcione a la administración tributaria informes, reportes con mercancías, datos, cifras, circunstancias 0 antecedentes falsos, incompletos, desfigurados o adulterados.

Art. 298 numeral 6 del Código Orgánico Integral Penal: Haga constar en las declaraciones tributarias datos falsos, incompletos, desfigurados o adulterados, siempre que el contribuyente no haya ejercido, dentro del año siguiente a la declaración, el derecho a presentar la declaración sustitutiva en la forma prevista en la ley.

Art. 298 numeral 7 del Código Orgánico Integral Penal. Falsifique o altere permisos, guías, facturas, actas, marcas, etiquetas o cualquier otro tipo de control de fabricación, consumo, transporte, importación y exportación de bienes gravados.

Art. 298 numeral 8 del Código Orgánico Integral Penal. Altere libros o registros informáticos de contabilidad, anotaciones, asientos u operaciones relativas a la actividad económica, así como el registro contable de cuentas, nombres, cantidades $o$ datos falsos. 
lustitia Socialis. Revista Arbitrada de Ciencias Jurídicas.

Año V. Vol. V. N³. Edición Especial. 2020-III:

Universidad Regional Autónoma de los Andes

Hecho el depósito de Ley: FA2016000064 ISSN: 2542-3371

FUNDACIÓN KOINONIA (F.K). Santa Ana de Coro, Venezuela

Paul Alejandro Centeno-Maldonado; Alex Carlos Ortega-Sinche; Williams Rafael Santillán-Abarca

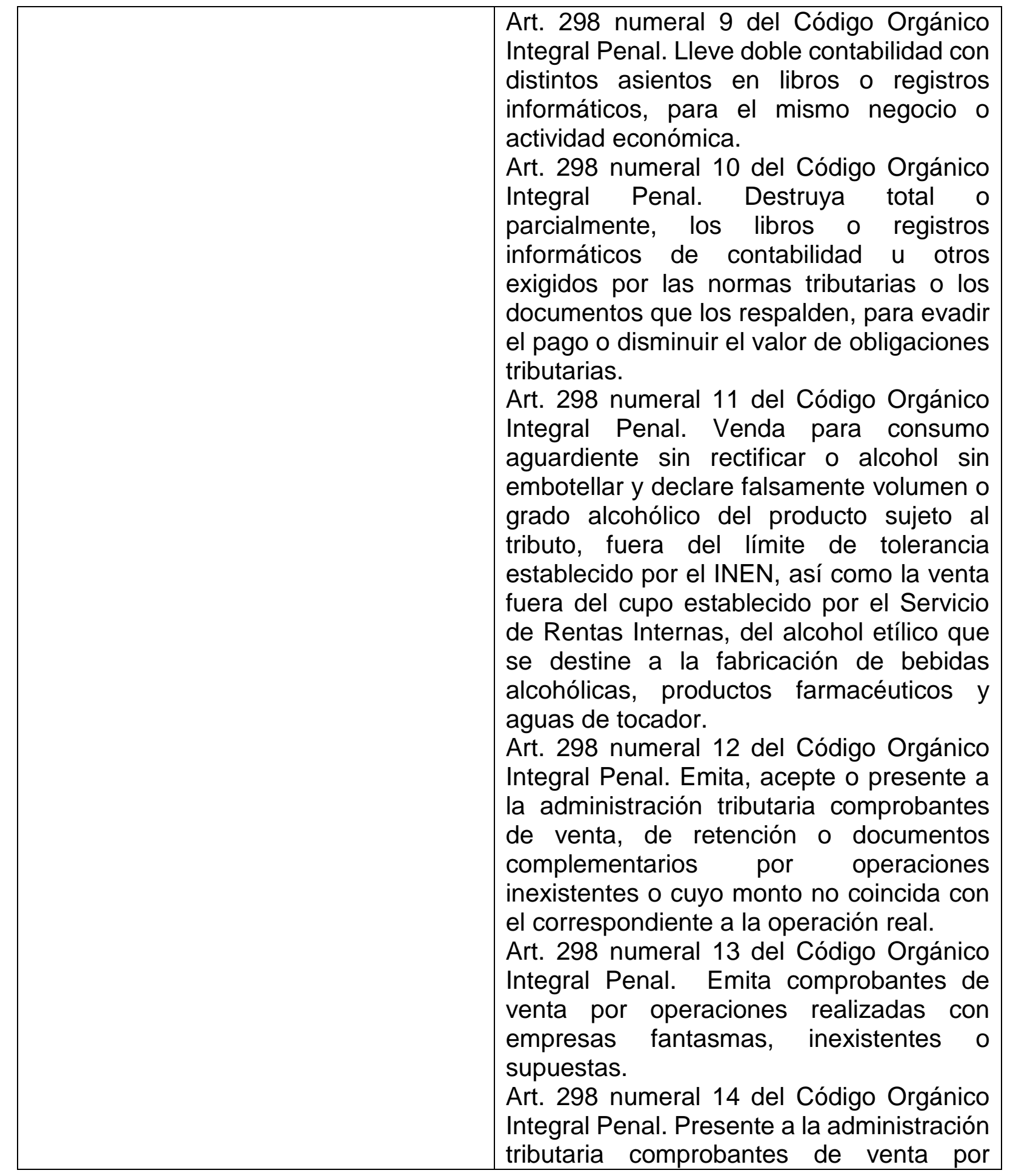


lustitia Socialis. Revista Arbitrada de Ciencias Jurídicas.

Año V. Vol. V. N³. Edición Especial. 2020-III:

Universidad Regional Autónoma de los Andes

Hecho el depósito de Ley: FA2016000064 ISSN: 2542-3371

FUNDACIÓN KOINONIA (F.K). Santa Ana de Coro, Venezuela

Paul Alejandro Centeno-Maldonado; Alex Carlos Ortega-Sinche; Williams Rafael Santillán-Abarca

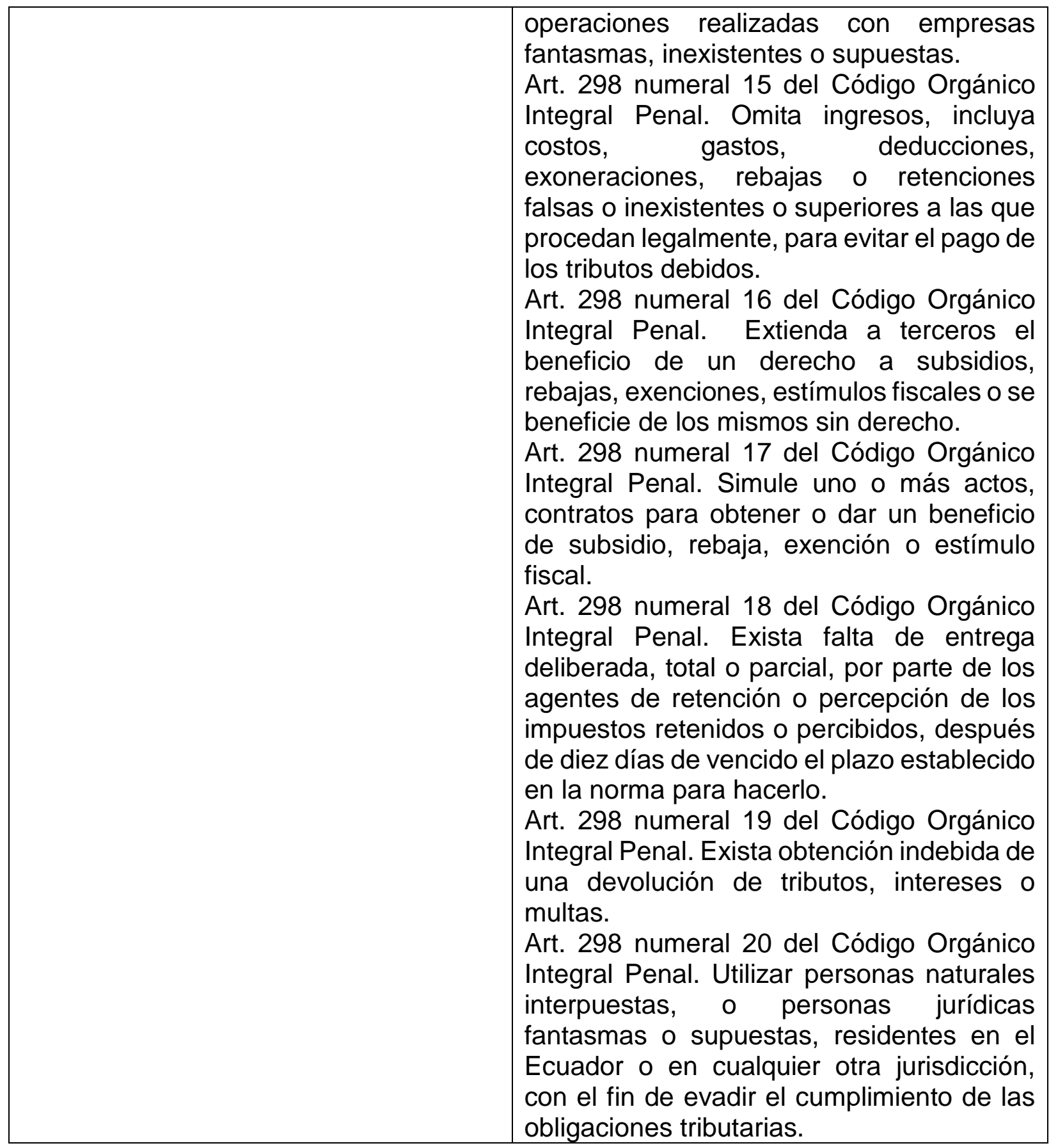


lustitia Socialis. Revista Arbitrada de Ciencias Jurídicas.

Año V. Vol. V. N³. Edición Especial. 2020-III:

Universidad Regional Autónoma de los Andes

Hecho el depósito de Ley: FA2016000064

ISSN: 2542-3371

FUNDACIÓN KOINONIA (F.K). Santa Ana de Coro, Venezuela

Paul Alejandro Centeno-Maldonado; Alex Carlos Ortega-Sinche; Williams Rafael Santillán-Abarca

Aunque se mantiene vigente el código tributario 2005, es evidente una ampliación en el Código Orgánico Integral Penal en 2014, sobre el proceder por parte de quien desarrolla una actividad económica, existiendo en el artículo 298, una serie de procedimientos y sanciones en favor de actuar ajustado al cumplimiento de la ley para la preservación de una conducta tributaria ética en razón de considerar la protección del patrimonio nacional.

\section{DISCUSIÓN}

Los resultados concuerdan con la postura de (Saltos-Orrala, 2017) y (Sánchez-Fonseca, et al., 2020), debido que a partir del 2014 lo relacionado a la tributación, se encuentra conforme a lo estipulado en el orden constitucional, evidenciándose que existe mejora en la configuración de un sistema de recaudación automatizado que coopera en la recolección de tributos, así como de crear una cultura para el pago de impuestos, sin embargo, es necesario profundizar en la educación tributaria para que la ciudadanía asuma con mayor responsabilidad en apego a las leyes, lo relacionado a la recaudación tributaria.

Por otro lado, se concuerda con (De-La-Torre-Lascano, 2017), (Chávez, 2015) у. (Sánchez-Huete, 2016), en destacar que el sistema es frágil en cuanto a los procesos aplicados para sancionar fuertemente a quienes incurran en delitos o fraudes fiscales, siendo considerable promover campañas de formación ciudadana sobre la diferencia entre delito y faltas en razón de confrontar y comprender tanto lo establecido en el código tributario y Código Orgánico Integral Penal en 2014, siendo posible establecer una concepción de mayor cumplimiento de la ley tributaria.

Se observa necesario reformar lo referido a la legislación tributaria del Ecuador, en relación de fortalecer desde lo sancionatorio y penal, las penas por incumplimiento de la ley, aunado a estabilizar el pago justo de las clases sociales más desfavorecidas, lo cual estaría en justa consonancia con el precepto constitucional del buen vivir, siendo esto similar a lo planteado por (Castañeda-Rodríguez, 2017), al referir que en América Latina donde se polariza la recaudación en detrimento del más vulnerable, siendo importante 
lustitia Socialis. Revista Arbitrada de Ciencias Jurídicas.

Año V. Vol. V. N³. Edición Especial. 2020-III:

Universidad Regional Autónoma de los Andes

Hecho el depósito de Ley: FA2016000064

ISSN: 2542-3371

FUNDACIÓN KOINONIA (F.K). Santa Ana de Coro, Venezuela

Paul Alejandro Centeno-Maldonado; Alex Carlos Ortega-Sinche; Williams Rafael Santillán-Abarca

considerar la aplicación de estrategias que incentiven la recaudación de los nuevos integrantes al sistema, no solo de los usuarios tradicionales al mismo.

En este sentido, (Alaña-Castillo, et al., 2018), destacan que en el Ecuador se desarrollan reformas tributarias que tienden a desinformar a los pequeños contribuyentes sobre el justo cumplimiento de sus obligaciones, por lo que concuerdan con (CastañedaRodríguez, 2017), en proponer estrategias para favorecer este segmento poblacional, siendo considerable crear ambientes de apoyo formativo en lo tributario y legal, en las universidades con la finalidad de contribuir a una cultura de recaudación favorable para la sociedad, lo cual a juicio de (Toledo-Zúñiga, 2018), se considera como necesaria la igualdad tributaria desde una reconstrucción de la jurisprudencia, siendo un tema a debatir por la comunidad jurídica del Ecuador con la finalidad de optimizar lo referido a la tributación en todos sus niveles.

\section{CONCLUSIÓN}

La normativa sancionatoria en materia de evasión tributara antes del Código Orgánico Integral Penal en 2014, se encontraba dispersa en diversos códigos, en la actualidad, existen dos modalidades de pena tributaria en el Ecuador, la primera de orden penal, calificada como delito, la segunda, como infracciones, faltas, tipificadas en el Código Tributario 2005, se evidencia que existe mejora en la configuración de un sistema de recaudación automatizado que coopera en la recolección de tributos, así como de crear una cultura para el pago de impuestos, sin embargo, el sistema es frágil en cuanto a los procesos aplicados para sancionar fuertemente a quienes incurran en delitos o fraudes fiscales, por lo tanto, se considera como necesaria la igualdad tributaria desde una reconstrucción de la jurisprudencia, siendo un tema a debatir por la comunidad jurídica del Ecuador con la finalidad de optimizar lo referido a la tributación en todos sus niveles. 
lustitia Socialis. Revista Arbitrada de Ciencias Jurídicas.

Año V. Vol. V. N³. Edición Especial. 2020-III:

Universidad Regional Autónoma de los Andes

Hecho el depósito de Ley: FA2016000064 ISSN: 2542-3371

FUNDACIÓN KOINONIA (F.K). Santa Ana de Coro, Venezuela

Paul Alejandro Centeno-Maldonado; Alex Carlos Ortega-Sinche; Williams Rafael Santillán-Abarca

\section{FINANCIAMIENTO}

No monetario.

\section{AGRADECIMIENTO}

A la Universidad Regional Autónoma de Los Andes; por motivar el desarrollo de la Investigación.

\section{REFERENCIAS CONSULTADAS}

Alaña-Castillo, T, Gonzaga-Añazco, S, \& Yánez-Sarmiento, M. (2018). Gestión tributaria a microempresarios mediante la creación del centro de asesoría caso: Umet sede Machala. [Tributary management for microentrepreneurs through the creation of the advice center case: Umet Branch Machala]. Revista Universidad $y$ Sociedad, 10(2), 84-88.

Castañeda-Rodríguez, V. (2017). La equidad del sistema tributario y su relación con la moral tributaria. Un estudio para América Latina. [The fairness of the tax system and its relationship with tax morality. A study for Latin America]. Investigación económica, 76(299), 125-152.

Chávez, L. (2015). Disertación sobre 'NIIF y Tributación: Impuestos Diferidos'. Ecuador. [Disertación sobre 'NIIF y Tributación: Impuestos Diferidos'. Ecuador]. Evento crecer, organizado por el Banco Mundial, IFAC y el Colegio de Contadores Públicos de Pichincha.

Código Orgánico Integral Penal. Registro Oficial № 180 - lunes 10 de febrero de 2014. Recuperado de https://n9.cl/g6sc

Código Tributario. (Codificación No. 2005-09). Recuperado de https://n9.cl/m8x30

De-La-Torre-Lascano, C. (2017). Relación existente entre paraísos fiscales, lavado de activos y defraudación tributaria. Un análisis desde la normativa de Ecuador Relación existente entre paraísos fiscales, lavado de activos y defraudación tributaria. Un análisis desde la normativa de Ecuador. [Existing relationship between tax havens, money laundering and tax defraud. An analysis from the norms of Ecuador]. Revista de la Facultad de Derecho, (43), 331. https://dx.doi.org/10.22187/rfd2017n2a2 
lustitia Socialis. Revista Arbitrada de Ciencias Jurídicas.

Año V. Vol. V. N³. Edición Especial. 2020-III:

Universidad Regional Autónoma de los Andes

Hecho el depósito de Ley: FA2016000064 ISSN: 2542-3371

FUNDACIÓN KOINONIA (F.K). Santa Ana de Coro, Venezuela

Paul Alejandro Centeno-Maldonado; Alex Carlos Ortega-Sinche; Williams Rafael Santillán-Abarca

Saltos-Orrala, M. (2017). Los Principios Generales del Derecho Tributario Según la Constitución de Ecuador. [The General Principles of Tax Law According to the Constitution of Ecuador]. Revista Empresarial, ICE-FEE-UCSG, 11(2), 61-67.

Sánchez-Fonseca, J, Esparza, I Gaibor, and M Barba. (2020). La evasión tributaria originada en el uso de comprobantes de venta. [Tax evasion originated in the use of sales receipts]. VI Congreso Internacional De La Ciencia, Tecnología, Emprendimiento E Innovación 2019, KnE Engineering, pages 149--163. DOI 10.18502/keg.v5i2.6231

Sánchez-Huete, M. A. (2016). La Administración Tributaria ante el delito. La nueva regularización voluntaria en QF. [La Administración Tributaria ante el delito. La nueva regularización voluntaria en QF]. № 7, abril, Navarra, Aranzadi, p. 39.

Toledo-Zúñiga, P. (2018). Igualdad tributaria: reconstrucción racional de la jurisprudencia constitucional española. [Tax Equity: A Rational Reconstruction of Spanish Constitutional Jurisprudence]. Revista de derecho (Valdivia), 31(1), 251271. https://dx.doi.org/10.4067/S0718-09502018000100251 